\title{
Topological Interference Management with Multiple Antennas
}

\author{
Hua Sun and Syed A. Jafar \\ Center for Pervasive Communications and Computing (CPCC) \\ University of California Irvine, Irvine, CA 92697
}

\begin{abstract}
The topological interference management problem refers to the study of the DoF of partially connected wireless communication networks with no channel state information at the transmitters (no CSIT) beyond the network topology, i.e., a knowledge of which channel coefficients are non-zero. While the problem is originally studied with single input sources and single output destinations (SISO), in this work we explore the implications of multiple inputs and multiple outputs (MIMO), highlighting fundamental differences and new phenomena.
\end{abstract}

\section{INTRODUCTION}

We are interested in the capacity of wireless communication networks with minimal CSIT, where the transmitters are only able to distinguish weak and significant channels, while having no further knowledge of the channel coefficients' realizations. It turns out that the core to such a capacity problem (e.g., constant gap approximation) is the first order (degrees of freedom, DoF) analysis of the underlying partially connected interference network (where the weak channels are set to zero) with no CSIT except the knowledge of the topology of the network. This is called the topological interference management (TIM) problem, as introduced in [1]. When all sources and destinations in the network are equipped with a single antenna, the TIM problem is shown in [1] to be essentially the index coding problem [2], which studies the optimal way to schedule the signal spaces to avoid interference based on only topology knowledge. TIM subsumes and generalizes conventional interference avoidance schemes such as TDMA/FDMA/CDMA.

Multiple antennas have a special place in the DoF studies of wireless networks, as the original setting that motivated the DoF metric, and the idea of spatial multiplexing, which became a stepping stone to the study of multiuser networks. As such, the implications of multiple-antenna nodes in our setting, i.e., partially connected wireless networks with no CSIT beyond network topology, are worth studying. The symmetric case where all nodes are equipped with the same number of antennas is studied in [1]. It turns out to be still essentially the index coding problem and the achievable DoF region through linear schemes is shown to include the scaled version of that of the SISO setting, i.e., spatial scale invariance property is satisfied. Also in [1], an asymmetric MIMO example is provided based on the idea of interference diversity, which is fundamentally distinct from the index coding problem. The example shows that the MIMO TIM problem is richer in

This work is supported by NSF grants CCF-1317351 and CCF-0963925. general and presents new challenges. The purpose of this work is to explore the MIMO TIM problem.

\section{SYSTEM MODEL}

Except for the assumption of multiple inputs and outputs, we will retain the framework of [1]. There are $S$ source nodes, labeled as $S_{1}, S_{2}, \cdots, S_{S}$ and $D$ destination nodes, labeled as $D_{1}, D_{2}, \cdots, D_{D}$. The channel input-output relationship is defined as

$$
Y_{i}(n)=\sum_{j=1}^{S} H_{i j} X_{j}(n)+Z_{i}(n), \forall i \in\{1,2, \ldots, D\}
$$

where at channel use index $n, Y_{i}(n) \in \mathbb{C}^{N_{i} \times 1}, X_{j}(n) \in$ $\mathbb{C}^{M_{j} \times 1}$ and $Z_{i}(n) \in \mathbb{C}^{N_{i} \times 1}$ are the signal observed at Destination $D_{i}$, the symbol sent from Source $S_{j}$ and the additive noise at Destination $D_{i}$, respectively. $Z_{i}(n)$ has zero mean and its covariance matrix is the $N_{i} \times N_{i}$ identity matrix. $H_{i j} \in \mathbb{C}^{N_{i} \times M_{j}}$ is the channel coefficient matrix between Source $S_{j}$ and Destination $D_{i}$, whose entries are drawn i.i.d from a continuous distribution and vary according to a block fading model with reasonably long coherence time. Source node $S_{j}$ has a set of independent messages, $\mathcal{W}\left(S_{j}\right)$, that it wants to send to their desired destinations. Destination node $D_{i}$ has a set of independent messages, $\mathcal{W}\left(D_{i}\right)$, that it desires. The set of all messages is denoted as

$$
\mathcal{W}=\cup_{i=1}^{D} \mathcal{W}\left(D_{i}\right)=\cup_{j=1}^{S} \mathcal{W}\left(S_{j}\right) .
$$

Each message has a unique source. Coding schemes, probability of error, achievable rates and capacity region are defined in the standard information theoretic sense. The achievable rate for message $W \in \mathcal{W}$ is denoted as $R(W)$. The average transmit power constraint at Source $j$ is set as $P_{j}$, i.e., $\mathbb{E}\left[\left\|X_{j}(n)\right\|^{2}\right] \leq P_{j}$, such that the following nominal interference-free rate guarantees are satisfied:

$$
\begin{aligned}
& \log \left(1+\frac{P_{j}}{M_{j}} \lambda_{\gamma}^{2}\left(H_{i j}\right)\right) \geq \log (1+\mathrm{SNR}) \\
& \forall \gamma \in\left\{1,2, \cdots, \min \left(M_{j}, N_{i}\right)\right\}, i \in[1: D], j \in[1: S] \\
& \mathcal{W}\left(D_{i}\right) \cap \mathcal{W}\left(S_{j}\right) \neq \phi
\end{aligned}
$$

where $\lambda_{\gamma}(H)$ is the $\gamma^{\text {th }}$ singular value of the channel matrix $H$. Thus, the power constraints are chosen such that, in the absence of all other messages, each message by itself (sent from Source $j$ to Destination $i$ ) can achieve a rate $\min \left(M_{j}, N_{i}\right) \log (1+\mathrm{SNR})$. 
Our interest is in minimal CSIT. Therefore, we assume the transmitters are only aware of the network topology information, represented by a topology matrix $\mathcal{T}=\left[t_{i j}\right]_{D \times S} \in$ $\{0,1\}_{D \times S}$, where $t_{i j}=0$ indicates that the channel matrix $H_{i j}$ is weak. The topology matrix $\mathcal{T}$ is available globally to all sources and destinations. For a first order (DoF) analysis we study a partially connected network where the weak channels are set to zero, i.e., if $t_{i j}=0$, then $H_{i j}=0$. In this partially connected model, we let SNR approach infinity (by increasing the transmit power for every source proportionately), and evaluate the achievable rates normalized by $\log$ SNR to find the DoF value,

$$
\operatorname{DoF}(W)=\lim _{\mathrm{SNR} \rightarrow \infty} \frac{R(W)}{\log (\mathrm{SNR})}, \forall W \in \mathcal{W} .
$$

The closure of the set of achievable DoF allocations for all messages is called the DoF region. The symmetric DoF value is defined as the largest value of DoF that is achievable by every message. We denote the DoF problem for the partially connected network as $\operatorname{TIM}(\mathcal{T}, \mathcal{W}(S), \mathcal{W}(D), \mathcal{M}(S), \mathcal{N}(D))$ where $\mathcal{M}(S) \triangleq\left[M_{1}, \cdots, M_{S}\right], \mathcal{N}(D) \triangleq\left[N_{1}, \cdots, N_{D}\right]$ are vectors denoting the transmit and receive antenna configurations, respectively. 1 represents the matrix (vector) with all entries equal to 1 , possibly with subscript indicating the dimension. Similar notation is used for $\mathbf{0} . \mathbf{I}_{R}$ denotes the $R \times R$ identity matrix. Throughout the paper, we will use the notion of linear schemes defined in detail in Section III of [1].

\section{RESULTS}

\section{A. SIMO TIM}

Here we study SIMO networks, which will highlight most of the distinctive aspects (gains, challenges and limitations) of MIMO TIM. In terms of interference alignment, without knowing the generic channel vectors at the sources, it may seem impossible at first sight, for alignment to take place at multiple receive antennas. The following example shows that alignment is indeed possible, i.e., there exists some space that is robust to the channel uncertainty due to the unknown SIMO channels.

Consider the 4-to-1 SIMO interference channel with a single antenna at each source and two antennas at each destination, as shown in Fig. 1.

We are interested in the symmetric DoF, denoted as $d$, for such a network. Suppose we use a linear scheme such that Source $j$ selects the beamforming matrix $\mathbf{V}_{j}$ to send the desired symbols for Destination $j$ over $T$ channel uses, where the size of $\mathbf{V}_{j}$ is $T \times T d$. The crux of the problem is how to consolidate the interference caused by Sources 2, 3, 4 at Destination 1 as much as possible under the channel uncertainty constraint. That is, without knowing the exact channel vectors, design $\mathbf{V}_{j}$ to minimize the size of the interference space of Destination 1,

$$
\begin{aligned}
\mathcal{I}_{1} & =\left[\begin{array}{lll}
H_{12} \otimes \mathbf{V}_{2} & H_{13} \otimes \mathbf{V}_{3} H_{14} \otimes \mathbf{V}_{4}
\end{array}\right] \\
& =\left[\begin{array}{lll}
h_{12} \mathbf{V}_{2} & h_{13} \mathbf{V}_{3} & h_{14} \mathbf{V}_{4} \\
h_{12}^{\prime} \mathbf{V}_{2} & h_{13}^{\prime} \mathbf{V}_{3} & h_{14}^{\prime} \mathbf{V}_{4}
\end{array}\right]
\end{aligned}
$$

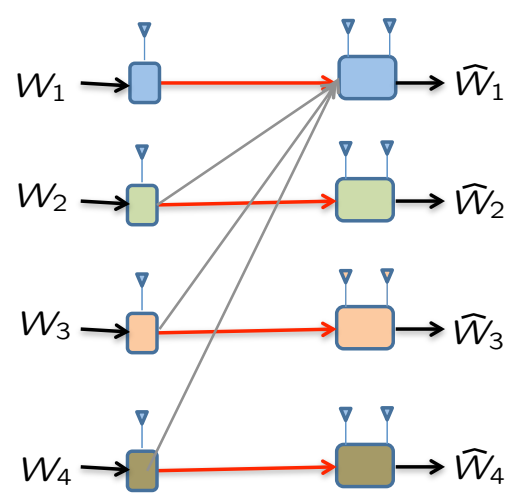

Fig. 1. The 4-to-1 $1 \times 2$ SIMO interference channel.

where $\otimes$ denotes the Kronecker product operation and the two elements of the channel vector $H_{i j}$ are denoted as $h_{i j}$ and $h_{i j}^{\prime}$, i.e., $H_{i j}=\left[h_{i j}, h_{i j}^{\prime}\right]^{T}$. When referring to the space of a matrix, we mean the span spanned by the columns of that matrix. As Destination 1 has two antennas while each source has only one antenna, the symbols sent from any two sources cannot align with each other at Destination 1, i.e.,

$$
H_{12} \otimes \mathbf{V}_{2} \cap H_{13} \otimes \mathbf{V}_{3}=\phi .
$$

Then the only way that alignment may take place is to let the space caused by interference from Source 4 to intersect with the union of the space spanned by interference from Sources 2 and 3, i.e.,

$$
\begin{array}{r}
\left(H_{12} \otimes \mathbf{V}_{2} \cup H_{13} \otimes \mathbf{V}_{3}\right) \cap H_{14} \otimes \mathbf{V}_{4} \neq \phi \\
\Rightarrow\left[\begin{array}{cc}
h_{12} \mathbf{V}_{2} & h_{13} \mathbf{V}_{3} \\
h_{12}^{\prime} \mathbf{V}_{2} & h_{13}^{\prime} \mathbf{V}_{3}
\end{array}\right] \cap\left[\begin{array}{c}
h_{14} \mathbf{V}_{4} \\
h_{14}^{\prime} \mathbf{V}_{4}
\end{array}\right] \neq \phi \\
\Rightarrow\left[\begin{array}{cc}
h_{12} \mathbf{I}_{T} & h_{13} \mathbf{I}_{T} \\
h_{12}^{\prime} \mathbf{I}_{T} & h_{13}^{\prime} \mathbf{I}_{T}
\end{array}\right]\left[\begin{array}{cc}
\mathbf{V}_{2} & \mathbf{0} \\
\mathbf{0} & \mathbf{V}_{3}
\end{array}\right] \cap\left[\begin{array}{c}
h_{14} \mathbf{V}_{4} \\
h_{14}^{\prime} \mathbf{V}_{4}
\end{array}\right] \neq \phi \\
\Rightarrow\left[\begin{array}{cc}
\mathbf{V}_{2} & \mathbf{0} \\
\mathbf{0} & \mathbf{V}_{3}
\end{array}\right] \cap\left[\begin{array}{cc}
h_{12} \mathbf{I}_{T} & h_{13} \mathbf{I}_{T} \\
h_{12}^{\prime} \mathbf{I}_{T} & h_{13}^{\prime} \mathbf{I}_{T}
\end{array}\right]\left[\begin{array}{c}
h_{14} \mathbf{V}_{4} \\
h_{14}^{\prime} \mathbf{V}_{4}
\end{array}\right] \neq \phi \\
\Rightarrow\left[\begin{array}{cc}
\mathbf{V}_{2} & \mathbf{0} \\
\mathbf{0} & \mathbf{V}_{3}
\end{array}\right] \cap\left[\begin{array}{c}
h \mathbf{V}_{4} \\
h^{\prime} \mathbf{V}_{4}
\end{array}\right] \neq \phi
\end{array}
$$

where (6) follows by multiplying the same full rank matrix from the left of both matrices and in (7), $h, h^{\prime}$ are rational functions of the involved interfering channels. From (7), we know that the interference space can be compressed if and only if $\mathbf{V}_{4}$ intersects with the intersection of $\mathbf{V}_{2}$ and $\mathbf{V}_{3}$, i.e.,

$$
\mathbf{V}_{2} \cap \mathbf{V}_{3} \cap \mathbf{V}_{4} \neq \phi .
$$

Note that this is strictly stronger than

$$
\mathbf{V}_{2} \cap \mathbf{V}_{4} \neq \phi, \mathbf{V}_{3} \cap \mathbf{V}_{4} \neq \phi .
$$

If we now set $\mathbf{V}_{2}=\mathbf{V}_{3}=\mathbf{V}_{4}=\mathbf{V}$ to maximally consolidate interference, then

$$
\mathcal{I}_{1}=\left[\begin{array}{ccc}
h_{12} \mathbf{V} & h_{13} \mathbf{V} & h_{14} \mathbf{V} \\
h_{12}^{\prime} \mathbf{V} & h_{13}^{\prime} \mathbf{V} & h_{14}^{\prime} \mathbf{V}
\end{array}\right] \subset\left[\begin{array}{cc}
\mathbf{V} & \mathbf{0} \\
\mathbf{0} & \mathbf{V}
\end{array}\right]
$$


as all columns of $\mathcal{I}_{1}$ belong to the span of $\mathbf{I}_{2} \otimes \mathbf{V}$. Then alignment is achieved across multiple receive antennas, although the sources do not know the realizations of the channel vectors. Note that this is also the best extent of alignment one can achieve. As such, one may conclude that as the interference space is confined into a space of dimension $2 T d$, as long as we choose $d$ such that the receive signal space is big enough to accommodate both the desired signal and aligned interference, i.e., $T d+2 T d \leq 2 T$, we can achieve the DoF value $2 / 3$, which is the same as the case with perfect CSIT [3], [4].

However, we have only considered the alignment of interference while neglecting the separability of desired signal from interference. In order to decode successfully, the desired signal space must be independent of the interference space. For this example, the signal space (union of the desired signal space $\mathcal{D}_{1}$ and the interference space $\mathcal{I}_{1}$ ) of Destination 1 is

$$
\operatorname{span}\left(\left[\begin{array}{ll}
\mathcal{D}_{1} & \mathcal{I}_{1}
\end{array}\right]\right)=\operatorname{span}\left(\left[\begin{array}{ccc}
h_{11} \mathbf{V}_{1} & \mathbf{V} & \mathbf{0} \\
h_{11}^{\prime} \mathbf{V}_{1} & \mathbf{0} & \mathbf{V}
\end{array}\right]\right)
$$

In order to ensure $\mathcal{D}_{1} \cap \mathcal{I}_{1}=\phi$, we have

$$
\begin{aligned}
{\left[\begin{array}{l}
h_{11} \mathbf{V}_{1} \\
h_{11}^{\prime} \mathbf{V}_{1}
\end{array}\right] \cap\left[\begin{array}{cc}
\mathbf{V} & \mathbf{0} \\
\mathbf{0} & \mathbf{V}
\end{array}\right] } & =\phi \\
\Rightarrow & {\left[\begin{array}{c}
\mathbf{V}_{1} \\
\mathbf{V}_{1}
\end{array}\right] \cap\left[\begin{array}{cc}
\frac{1}{h_{11}} \mathbf{V} & \mathbf{0} \\
\mathbf{0} & \frac{1}{h_{11}^{\prime}} \mathbf{V}
\end{array}\right]=\phi }
\end{aligned}
$$

where (11) follows by scaling the rows of both matrices by the same factor. From (11), we know $\mathbf{V}_{1} \cap \mathbf{V}=\phi$, which means that the independence condition of desired signal from aligned interference must hold over each receive antenna. Note that this is distinct from the perfect CSIT case, where resolvability only needs to be satisfied in the total receive signal space of all receive antennas [5], [6]. From this constraint, we know that the aligned signal space must have dimension no more than $T(1-d)$, i.e., $|\mathbf{V}| \leq T(1-d)$, where $|\mathbf{V}|$ denotes the number of columns in $\mathbf{V}$. Then Sources $2,3,4$ are each left with a $T[d-(1-d)]=T(2 d-1)$ dimensional space that should be designed such that they do not align at Destination 1. We denote these spaces as $\mathbf{U}_{i}, i \in\{2,3,4\}$ such that $\mathbf{V}_{i}=$ $\left[\begin{array}{ll}\mathbf{V} & \mathbf{U}_{i}\end{array}\right]$ and the signal space at Destination 1 is then

$\operatorname{span}\left(\left[\begin{array}{cccccc}h_{11} \mathbf{V}_{1} & \mathbf{V} & 0 & h_{12} \mathbf{U}_{2} & h_{13} \mathbf{U}_{3} & h_{14} \mathbf{U}_{4} \\ h_{11}^{\prime} \mathbf{V}_{1} & 0 & \mathbf{V} & h_{12}^{\prime} \mathbf{U}_{2} & h_{13}^{\prime} \mathbf{U}_{3} & h_{14}^{\prime} \mathbf{U}_{4}\end{array}\right]\right)$

To guarantee decodability, the number of columns in the above matrix must be no more than the size of the total receive signal space, i.e.,

$$
\begin{aligned}
& \left|\mathbf{V}_{1}\right|+2|\mathbf{V}|+\left|\mathbf{U}_{2}\right|+\left|\mathbf{U}_{3}\right|+\left|\mathbf{U}_{4}\right| \leq 2 T \\
\Rightarrow \quad & d+2(1-d)+3(2 d-1) \leq 2 \\
\Rightarrow & d \leq 3 / 5 .
\end{aligned}
$$

Thus, we arrive at the upper bound on the symmetric DoF achieved by linear schemes.

This linear outer bound derivation also guides us to the achievable scheme. To achieve $3 / 5$ DoF per user, each source sends 3 symbols to its desired destination, over $T=5$ channel uses. By the insights from the outer bound, $\mathbf{V}_{i}, i \in\{2,3,4\}$ is divided into an aligned part and a non-aligned part, namely, $\mathbf{V}_{i}=\left[\begin{array}{ll}\mathbf{V} & \mathbf{U}_{i}\end{array}\right]$, where $\mathbf{V}$ is a $5 \times 2$ matrix and $\mathbf{U}_{i}$ are three $5 \times 1$ vectors. Furthermore, we choose them as follows

$\mathbf{V}=\left[\begin{array}{ll}1 & 0 \\ 0 & 1 \\ 0 & 0 \\ 0 & 0 \\ 0 & 0\end{array}\right], \mathbf{U}_{2}=\left[\begin{array}{l}0 \\ 0 \\ 1 \\ 0 \\ 0\end{array}\right], \mathbf{U}_{3}=\left[\begin{array}{l}0 \\ 0 \\ 0 \\ 1 \\ 0\end{array}\right], \mathbf{U}_{4}=\left[\begin{array}{l}0 \\ 0 \\ 0 \\ 0 \\ 1\end{array}\right]$

Finally, $\mathbf{V}_{1}=\left[\mathbf{U}_{2}, \mathbf{U}_{3}, \mathbf{U}_{4}\right]$. As such, it is easily seen that the signal space matrix (12) has full rank almost surely such that Destination 1 can decode its desired message. Decodability at other destinations is trivially satisfied.

We conclude that the linear symmetric DoF value for the 4to- $11 \times 2$ SIMO TIM problem is $3 / 5$. In this case, the highest DoF value that can be achieved by using single receive antenna only (treating as a SISO instance and deploying the index coding solution) and multicast are both $1 / 2$. Note that setting $|\mathbf{V}|=0$ (no alignment) gives us the multicast solution and fixing $\left|\mathbf{U}_{i}\right|=0$ reduces to the SISO setting where decoding over one antenna suffices. This shows that to obtain the best linear DoF, we have to jointly optimize and allocate the spaces balancing between alignment (which saves interference space but introduces additional separability constraint over each receive antenna) and multicast (which does not compress interference but separability needs to hold only within the total receive space).

To summarize the structure of the SIMO TIM problem, while alignment still happens in the same way as the SISO case, a new separability condition emerges. Apart from the usual requirement that the size of the union of the desired and interfering spaces should be no more than the size of the total receiver space, we have an extra condition, which says that the aligned interference must be independent of the desired signal over each receive antenna. This constraint severely limits the achievable linear DoF in the TIM setting (no CSIT) if we compare with the setting with perfect CSIT where the perantenna separability constraint is not present.

For example, let us consider a SIMO TIM instance where a destination which has $R$ receive antennas and desires one message is faced by $K(\geq R)$ interfering sources, each with an independent message for its desired destination. The signal space used by each interferer can be divided into the aligned and non-aligned part, with size $d_{1}$ and $d_{2}$ each. At the considered destination, the aligned part may overlap and the non-aligned part would remain independent. Due to symmetry, we can assume $d_{1}$ and $d_{2}$ are the same for all interferers. Note that $d_{1}$ and $d_{2}$ are design variables. Then according to the separability conditions stated above, we have

$$
\begin{aligned}
d_{1}+d_{2} & =d \\
d+d_{1} & \leq 1 \\
d+R d_{1}+K d_{2} & \leq R
\end{aligned}
$$

where (17) follows from the per-antenna separability constraint and alignment can only take place in a tensor product space 
$\mathbf{I}_{R} \otimes \mathbf{V}$ where $|\mathbf{V}|$ must be larger than $d_{1}$ to accommodate each individual interference. In the overall separability constraint (18), $d$ is the size of the desired signal, $R d_{1}$ is the minimum size possible for the aligned interference as the destination has $R$ antennas while each source has only 1 antenna and $K d_{2}$ is the size of the non-aligned interference. We want to maximize $d$ subject to these constraints, from which we get

$$
d \leq \frac{K}{2 K-R+1} .
$$

As such, regardless of how we allocate $d_{1}$ and $d_{2}$, with more interferers (large $K$ ), the linear DoF bound goes to $1 / 2$ as $K$ becomes large, i.e., the gain of multiple receive antennas vanishes. This is in sharp contrast with the perfect CSIT setting as well [3].

While the insights that emerge from the analysis of linear schemes seem more broadly applicable, we show next that they can also be translated into information theoretic arguments. Towards this end, we prove that symmetric information theoretic (not restricted to linear) DoF value of the 4-to- $1 \times 2$ SIMO TIM problem is $3 / 5$. This is stated in the following theorem.

Theorem 1: The symmetric DoF of the 4-to- $11 \times 2$ SIMO TIM problem is $\frac{3}{5}$.

Proof: As the achievability is already shown above, we only need to prove that $3 / 5$ is an information theoretic outer bound. For such a purpose, we first do a change of basis operation at each destination such that only the first antenna receives the desired signal. As such, we have

$$
\begin{aligned}
& Y_{1}=\left[\begin{array}{l}
y_{1} \\
y_{1}^{\prime}
\end{array}\right]=\left[\begin{array}{c}
h_{11} X_{1}+h_{12} X_{2}+h_{13} X_{3}+h_{14} X_{4}+Z_{1} \\
h_{12}^{\prime} X_{2}+h_{13}^{\prime} X_{3}+h_{14}^{\prime} X_{4}+Z_{1}^{\prime}
\end{array}\right] \\
& Y_{i}=\left[\begin{array}{l}
y_{i} \\
y_{i}^{\prime}
\end{array}\right]=\left[\begin{array}{c}
h_{i i} X_{i}+Z_{i} \\
Z_{i}^{\prime}
\end{array}\right], \quad i \in\{2,3,4\} .
\end{aligned}
$$

Also define

$$
L_{j}=g_{j 2} X_{2}+g_{j 3} X_{3}+g_{j 4} X_{4}+Z_{j}, j \in\{1,2,3,4,5\}
$$

to be generic combinations of $X_{2}, X_{3}$ and $X_{4}$ plus independent noise, where $g_{j i}, i \in\{2,3,4\}$ are drawn independently from the same distribution as each of the channel coefficients and $Z_{j} \sim \mathcal{N}(0,1)$. Now consider codewords spanning $N$ channel uses. For Destination 1, from Fano's inequality, we have

$$
\begin{aligned}
N\left(R_{1}-\epsilon\right) & \leq I\left(W_{1} ; Y_{1}\right)=I\left(W_{1} ; y_{1}^{N}, y_{1}^{N^{\prime}}\right) \stackrel{(a)}{=} I\left(W_{1} ; y_{1}^{N} \mid y_{1}^{N^{\prime}}\right) \\
& =h\left(y_{1}^{N} \mid y_{1}^{N^{\prime}}\right)-h\left(y_{1}^{N} \mid y_{1}^{N^{\prime}}, W_{1}\right) \\
& \leq N[\log P+o(\log P)]-h\left(L_{1}^{N} \mid L_{2}^{N}\right)
\end{aligned}
$$

where $(a)$ follows from the independence of $W_{1}$ and $y_{1}^{\prime}$. In (20), $P \triangleq \sum_{i=1}^{4} P_{i}$, the first term is due to the fact that the differential entropy of a random variable is maximized by Gaussian distribution with the same variance and the second term follows from the statistical equivalence between $\left(y_{1}-h_{11} X_{1}, y_{1}^{\prime}\right)$ and $\left(L_{1}, L_{2}\right)$. Such statistical equivalence argument has also been used in [7], [8], [9]. For Destination $i \in\{2,3,4\}$, we have

$$
\begin{aligned}
N\left(R_{i}-\epsilon\right) & \leq I\left(W_{i} ; Y_{i}\right)=I\left(W_{i} ; y_{i}^{N}, y_{i}^{N^{\prime}}\right)=I\left(W_{i} ; y_{i}^{N} \mid y_{i}^{N^{\prime}}\right) \\
& =h\left(y_{i}^{N} \mid y_{i}^{N^{\prime}}\right)-h\left(y_{i}^{N} \mid y_{i}^{N^{\prime}}, W_{i}\right) \\
& \leq h\left(y_{i}^{N}\right)+N o(\log P)
\end{aligned}
$$

Adding (21) for $i \in\{2,3,4\}$, we have

$$
\begin{aligned}
& N\left(R_{2}+R_{3}+R_{4}-\epsilon\right) \\
\leq & h\left(y_{2}^{N}, y_{3}^{N}, y_{4}^{N}\right)+N o(\log P) \\
= & h\left(L_{3}^{N}, L_{4}^{N}, L_{5}^{N}\right)+N o(\log P) \\
\leq & h\left(L_{3}^{N}\right)+h\left(L_{4}^{N} \mid L_{3}^{N}\right)+h\left(L_{5}^{N} \mid L_{3}^{N}\right)+N o(\log P) \\
\leq & N \log (P)+2 h\left(L_{1}^{N} \mid L_{2}^{N}\right)+N o(\log P)
\end{aligned}
$$

where (22) follows from the independence between $y_{2}, y_{3}$ and $y_{4}$. (23) follows from the transform matrix from $\left(y_{2}, y_{3}, y_{4}\right)$ to $\left(L_{3}, L_{4}, L_{5}\right)$ has bounded determinant and does not scale with SNR. (25) is due to the fact that $L_{j}, j \in\{1, \ldots, 5\}$ have the same distribution.

Adding 2 times (20) with (25), normalizing it by $N$ and letting $N$ approaches infinity, we have

$$
2 R_{1}+R_{2}+R_{3}+R_{4} \leq 3 \log (P)+o(\log P) .
$$

Setting $R_{1}, R_{2}, R_{3}, R_{4}$ to be equal, normalizing by $\log (\mathrm{SNR})$ and letting SNR approaches infinity will complete the proof.

The presented arguments can also be extended to establish the symmetric DoF for the class of SIMO TIM problem $\operatorname{TIM}\left(\mathcal{T}, \mathcal{W}(S), \mathcal{W}(D), \mathbf{1}_{S}, \mathcal{N}(D)\right)$ where the corresponding SISO version $\operatorname{TIM}\left(\mathcal{T}, \mathcal{W}(S), \mathcal{W}(D), \mathbf{1}_{S}, \mathbf{1}_{D}\right)$ is half-ratefeasible. This class is fully characterized in [10], [11], [1]. This includes the many-to-one SIMO TIM problem as a special case. Details will be presented in the full paper (currently under preparation).

Finally, it is interesting to compare the SIMO TIM problem with the index coding problem. If we want to draw some similarities, then a new variant of index coding may be defined. Recall that the SISO TIM problem is linearly equivalent to the index coding problem, which can be understood as follows. In the SISO TIM problem, destination $D_{i}$ observes the signal $Y_{i}=\sum_{j=1, j: t_{i j} \neq 0}^{S} H_{i j} X_{j}+Z_{i}$, where all variables are scalars. As linear encoding and decoding are employed, the exact channel coefficients do not matter, we can assume all channels are 1, without loss of generality. As such, we can assume each destination receives the same signal $B=\sum_{j=1}^{S} X_{j}+Z_{i}$ and Destination $D_{i}$ is aware of the messages $\mathcal{W}\left(S_{j}\right), \forall j: t_{i j}=0$ to reconstruct and cancel the non-connected transmit signals. Now this is equivalent to an index coding problem where $B$ is the bottleneck signal that is available to all destinations and the known messages are the antidotes. We proceed to the SIMO case where Destination $i$ observes $Y_{i}=\sum_{j=1, j: t_{i j} \neq 0}^{S} H_{i j} X_{j}+Z_{i}$. Note that $H_{i j}$ is a vector now. Consider the situation where $N_{i}$ are equal for all $i$. As the channels are all i.i.d and capacity only depends on marginals, we can also assume all the destinations still observe the same signal, $B=\sum_{j=1}^{S} H_{j} X_{j}+Z_{i}$, where $H_{j}$ 
is an $N_{i} \times 1$ vector with the same distribution as $H_{i j}$. Also Destination $D_{i}$ is aware of the messages $\mathcal{W}\left(S_{j}\right), \forall j: t_{i j}=0$. Now the difference from the index coding problem is obvious. In such a SIMO TIM problem, each destination sees the same bottleneck signal, accompanied by some side information consisted of messages from unconnected sources. As opposed to the original index coding setting where the bottleneck signal is a sum of the transmitted signals, here the bottleneck signal is $N_{i}$ generic linear combinations of the transmitted signals. So confined to linear schemes, the SIMO TIM problem is a generalized form of index coding problem where every destination still receives the same bootlick signal, albeit with a special form, i.e., it is several generic linear combinations of the same transmitted symbols. The situation where different destinations have different number of antennas is similar. This can be viewed as a layered version of index coding, where the destinations with the same number of receive antennas see the same bottleneck signals (still generic combinations of transmitted symbols) and the destinations with more antennas see more generic linear combinations plus those received by the destinations with fewer number of antennas. Given this distinct property, it is not clear how to generalize the previous index coding solution here.

\section{B. Solely Adding Transmit Antennas is Not Useful}

Given an arbitrary SISO TIM instance, we also explore the implications of adding transmit antennas, i.e., the MISO setting. Without knowing the exact channel realizations, equipping with more transmit antennas at the sources seems useless. This intuition is verified from information theoretic perspective. The result is stated in the following theorem.

Theorem 2: The DoF region of the MISO TIM problem $\operatorname{TIM}\left(\mathcal{T}, \mathcal{W}(S), \mathcal{W}(D), \mathcal{M}(S), \mathbf{1}_{D}\right) \quad$ is the same as the DoF region of the SISO TIM problem $\operatorname{TIM}\left(\mathcal{T}, \mathcal{W}(S), \mathcal{W}(D), \mathbf{1}_{S}, \mathbf{1}_{D}\right)$

Proof: The DoF region of the SISO case is trivially contained within that of the MISO case. We show that the other direction also holds, by transforming any MISO instance into the corresponding SISO instance, without loss of DoF during the transformation. Since each destination must decode its desired messages based solely on its own received signal, the DoF region only depends on the marginal distributions. Consider the channel vectors from Source $j$ to its connected destinations, i.e., destinations $i: t_{i j}=1$, as the channels are spatially i.i.d, without loss of generality let us make the channel vector for one of the destinations (say, destination $\bar{i}$ and $t_{\bar{i} j}=1$ ), also the channel vector of all connected destinations, i.e., $H_{i j}=H_{\bar{i} j}, \forall i: t_{i j}=1$. Now the overall channel matrix from Source $j$ to all connected destinations has only unit rank, the source can do a change of basis operation to discard all redundant dimensions, leaving Source $j$ with a single antenna. Repeating this argument for all sources gives us the desired SISO instance, which completes the proof.

As the SISO TIM problem is essentially the index coding problem and Theorem 2 establishes the equivalence between
MISO and SISO TIM, the MISO TIM problem still does not go beyond index coding.

The above theorem extends easily to the case where there are more transmit than receive antennas. As such, we conclude that it is useless for a source to have more transmit than each of its connected destinations' receive antennas. In other words, without loss of DoF, for any TIM instance, for any Source $j$, we can always reduce the number of transmit antennas from $M_{j}$ to $\max \left(N_{i}\right), i: t_{i j}=1$, whenever $M_{j} \geq N_{i}, \forall i: t_{i j}=1$. As such, without any loss, we can exclusively focus on the cases where there are fewer transmit than receive antennas.

\section{CONCLUSION}

The implications of MIMO are explored for the topological interference management problem. The extension goes beyond index coding and reveals new opportunities as well as distinct challenges. Specifically, we show that having more transmit than receive antennas is not helpful. A general MIMO TIM instance can be treated as a SIMO TIM instance where the transmit antennas are decomposed. For the SIMO TIM problem, while alignment takes place very much like a SISO setting, distinct separability conditions emerge, where aligned interference needs to be independent of the desired signal over each receive antenna. This places fundamental limits on using multiple antennas in the TIM setting.

\section{REFERENCES}

[1] S. A. Jafar, "Topological Interference Management through Index Coding," IEEE Transactions on Information Theory, vol. 60, no. 1, pp. "529568", Jan. 2014.

[2] Z. Bar-Yossef and Y. Birk and T. S. Jayram and T. Kol, "Index Coding With Side Information," IEEE Trans. on Information Theory, vol. 57, no. 3, pp. 1479 - 1494, March 2011.

[3] T. Gou and S. Jafar, "Degrees of freedom of the K user M $\times$ N MIMO interference channel," IEEE Trans. on Information Theory, vol. 56, no. 12, pp. 6040-6057, December 2010.

[4] A. Ghasemi, A. Motahari, and A. Khandani, "Interference alignment for the K user MIMO interference channel," in Proceedings of International Symposium on Information Theory (ISIT). IEEE, 2010, pp. 360-364.

[5] H. Sun, T. Gou, and S. Jafar, "Degrees of freedom of MIMO X networks: Spatial scale invariance, one-sided decomposability and linear feasibility," IEEE Transactions on Information Theory, vol. 59, no. 12, pp. "8377-8385", Dec. 2013.

[6] M. Zamanighomi and Z. Wang, "Multiple-antenna interference channels with real interference alignment and receive antenna joint processing," ArXiv: http://arxiv.org/abs/1301.6315, 2013, submitted to the IEEE Trans. on Information Theory.

[7] C. Huang, S. A. Jafar, S. Shamai, and S. Vishwanath, "On Degrees of Freedom Region of MIMO Networks without Channel State Information at Transmitters," IEEE Transactions on Information Theory, no. 2, pp. 849-857, Feb. 2012.

[8] Y. Zhu and D. Guo, "The degrees of freedom of isotropic mimo interference channels without state information at transmitters,' Information Theory, IEEE Transactions on, no. 1, pp. 341-352, Jan. 2012.

[9] C. S. Vaze and M. K. Varanasi, "A new outer-bound via interference localization and the degrees of freedom regions of mimo interference networks with no csit," in print, IEEE Trans. on Information Theory, Available: http://arxiv.org/abs/1105.6033.

[10] A. Blasiak, R. Kleinberg, and E. Lubetzky, "Index coding via linear programming," ArXiv:1004.1379, April 2010. [Online]. Available: http://www.cs.cornell.edu/ ablasiak/papers/bkl-beta.pdf

[11] H. Maleki, V. Cadambe, and S. Jafar, "Index coding - an interference alignment perspective," ISIT 2012, Preprint of Full Paper available at ArXiv:1205.1483, 2012. 